\title{
A new algorithm for smart cards
}

\author{
Ch. Marco, P. Morillo*
}

Dep. de Matemática Aplicada y Telemática, E.T.S.E.T.B.

Universidad Politécnica de Cataluña

C/ Gran Capitán s/n, módulo C3

Barcelona (España)

Tel. (34) (3) 4016538

E-mail:cmarco@mat.upc.es

*Tel. (34) (3) 4016008

*E-mail:paz@mat.upc.es

Fax (34) (3) 4015981

\begin{abstract}
In this paper we present a new cryptographic scheme based on the discrete logarithm problem which allows to perform encryption, authentication and digital signatures. Due to its versatility and efficiency it can be appropriated for the identification and exchange of information between smart cards and between smart cards and terminals.
\end{abstract}

Cryptography, discrete logarithm, smart card

\section{INTRODUCTION}

At the present, a lot of security services, many of them based on cryptographic systems, are invading our every day life. The limitations of conventional criptography have been already overpassed, since public key cryptography provided the solution to the two basic problems of the secret key cryptography: the secure exchange of secret keys and the possibility to generate electronic signatures.

We can say that, since 1976, when the concept of public key cryptography was presented by Diffie and Hellman in (Diffie-Hellman, 1976), public key cryptosystems has shown to be ideal for an open communications world. Typical applications of public key 
cryptography, such as identification, access control, exchange and/or storage of enciphered data, software protection, etc... are commonly used in our days, and their use in the future seems to be unlimited.

According to this criteria, it is not very intrepid to think that smart cards can play a very important role in the world of security. In fact, it seems that smart cards will become the basic security device in the future due to three fundamental reasons:

1. As it is well known, a smart card is able to store and process information and, therefore, to perform cryptographic algorithms. We can say that a smart card is a true microcomputer.

2. Smart cards are very easy to use (the user only must remember his own secret key to authenticate himself against the card) and, moreover, they are roughly of the size of common credit cards.

3. Smart cards offer both logical and physical security.

Nevertheless, in spite of the continuous evolution of the technology, the limitations of the cards in terms of memory capacity and computational power must be taken into account when designing cryptosystems for smart cards. According to this idea, Schnorr in (Schnorr, 1991) proposed an algorithm which minimizes the length of the keys and Fiat and Shamir in (Fiat-Shamir, 1987) proposed an algorithm which minimizes the number of operations.

In this paper we propose a new cryptographic protocol which is based on the ElGamal encryption algorithm (ElGamal, 1985), so it is based on the discrete logarithm problem. Our algorithm includes identification, encryption with message authentication, and signature generation. The most important feature of our scheme is its flexibility since it provides different security functions. Due to its characteristics the algorithm can be considered very suitable for interactions between smart cards and terminals. As we will show, it can be used to authenticate the terminal or the cards, to exchange confidential and authenticated messages and to generate and verify digital signatures. We will also show that the new scheme can be a good alternative when choosing a cryptographic algorithm for smart cards.

\section{THE NEW SCHEME}

We assume the existence of a trusted authority (a government, a credit card company, a computer center, a military headquarters, etc.) which issues the smart cards to the users.

The cards contain an identification number generated by the KAC (Key Authentication Center), $I$, and a signature for the pair (I, v), where $v$ is the user public key. The pair $(I, v)$ allows to identify the card. The identification number and the public key of the card have to be verified by the terminal (or other card) reading I and $\mathbf{v}$ from a public file or checking the KAC's signature using the KAC's public key. So, any interaction between a card and a terminal or between two cards which requires the use of their respective public keys must contain a previous verification of such keys.

On the other hand, we have just mentioned that the new scheme is based on the discrete logarithm problem in a subgroup of $Z_{p}^{*}$ (McCurley, 1990) (Odlyzko, 1985). So, a modulus prime $\mathrm{p}$, a prime $\mathrm{q}$ which divides $\mathrm{p}-1$, and an integer $\mathrm{b}$, are required. The 
private and public keys are the integers $s$ modulo $q$ and $v=b^{-s} \bmod p$, respectively. Also a one-way hash function, $h$, is used.

\subsection{General scheme: Encryption with authentication and signature generation}

The algorithm follows the next steps:

1. A picks a random integer $0<r<q$.

2. A calculates $z=b^{r} \bmod p$.

3. A calculates $x=m \cdot v_{B}^{r .} e^{\bmod q} \bmod p$, where the message, $m$, is an integer, $0<m<p$, which includes at a minimum:

- The identity of A and B, to show that the message was generated by A and intended for $B$.

- The information, $\mathrm{m}_{1}$.

- A timestamp, $t_{A}$, including the generation and expiration time to prevent delayed delivery of messages.

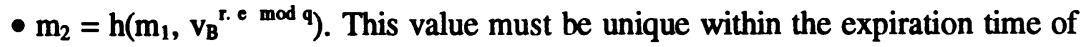
the message. Then, the user $B$ can store it until the message expires and reject messages with the same value $m_{2}$ which are received during that period.

The value $e$ is calculated as $\mathrm{e}=\mathrm{h}\left(\mathrm{m}_{1}, \mathrm{z}\right)$.

4. A calculates $y$ such that $s_{A} \cdot y=-(r \cdot e+1) \bmod q$.

5. A sends to $B:(x, y, z)$.

Message reception and signature verification:

6. B deciphers calculating $m=x \cdot v_{B} \cdot v_{A}{ }^{y \cdot s B} \bmod q \bmod p$.

7. $B$ authenticates the message checking that $m_{2}=h\left(m_{1}, x / m \bmod p\right)=h\left(m_{1}, v_{B}^{r . e} \bmod q\right.$ $\bmod$ p).

8. The signature is verified checking that $\left(z^{-e .} v_{A}^{y}\right) \bmod p=b$.

One special and important feature of the proposed scheme is that encryption includes authentication. This is a basic difference with respect to the general encryption algorithms since it makes possible to authenticate a message independently of the signature generation, and simultaneously to the encryption.

The receiver doesn't have to check the signature to verify the authenticity of the message. Such verification is performed very easily after deciphering (the redundant information included in the message, $\mathrm{m}$, allows the verification of the authenticity of the message). Signature verifications (if the message is signed) would be performed only if later authentications were necessary.

Finally, the new scheme not only allows to encrypt and authenticate messages and/or generate electronic signatures. With slight variations the algorithm can be also used as an identification protocol. The identification can be performed with two different procedures which are based on the encryption and authentication scheme and on the signature scheme, respectively. Both options, denoted as one-way and two-way identification schemes, are presented. 


\subsection{One-way identification}

1. A picks a random integer $r, 0<r<q$.

2. A builds a messsage, $m$, such as it has been explained before, but using a random value $\mathrm{m}_{1}$.

3. A calculates $x=m \cdot v_{B}{ }^{\text {re } \bmod q} \bmod p$, where e can be a random integer in $\left\{1, \ldots, 2^{t}-1\right\}$ or simply e $=1$.

4. A calculates $y$ such that $s_{A} \cdot y=-(r \cdot e+1) \bmod q$.

5. A sends to $B:(x, y)$.

6. $B$ calculates $m=x \cdot v_{B} \cdot v_{A}{ }^{y \cdot s B \bmod q} \bmod p$ and checks that $m_{2}=h\left(m_{1}, x / m\right)$.

\subsection{Two-way identification}

1. B sends a random integer, $\mathrm{k}, 0<\mathrm{k}<\mathrm{q}$ (or with shorter length).

2. A picks a random integer $0<r<q$.

3. A calculates $z=b^{r} \bmod p$.

4. A calculates y such that $s_{A} \cdot y=-(r \cdot e+1) \bmod q$, with $e=h(k, z)$.

5. A sends to $B$ : $(z, y)$.

6. $B$ calculates $e^{\prime}=h(k, z)$.

7. $B$ checks that $\left(z^{-e^{\prime}} \cdot v_{A}^{y}\right) \bmod p=b$.

\section{SECURITY}

The security of the new protocol lies on the difficulty to calculate discrete logarithms. The identification and signature protocols and the encryption algorithm, only can be broken if the attacker gets the secret keys.

So, let's see how the algorithm can be attacked.

Including $m_{2}=h\left(m_{1}, v_{B}{ }^{r . e} \bmod q\right.$ mod $\left.p\right)$ we avoid known plaintext attacks since $m_{2}$, which contains random information, is never made public. An attacker can't obtain a pair plaintext-ciphertext.

To supplant A, a forger can't use an old one-way identification message of A since its contents would reveal to $B$ that it is a false message. Moreover, without the secret key of $B$, an attacker is unable to understand a message from $A$ and, therefore, he can't obtain any information.

To falsify a signature of $A$ (or supplant $A$ in the two-way identification procedure), a forger could fix $y$, and then try to find $z$ such that $z^{-t} \cdot v_{A}^{y} \bmod p=b$, with $e$ $=h\left(m_{1}, z\right)($ or $h(k, z))$, or he could fix $z$, and try to calculate $y$. In both cases the problem is very hard.

Both the authentication and signature schemes, apparently, don't reveal any information about the user secret key, since all values are calculated using random variables. Therefore, the enciphered messages always depend on a random number. 
So, we can conclude indicating that we have not found any efficient attack to the new scheme.

\section{COMPLEXITY}

In order to show the validity of the new scheme it is interesting to compare it with two schemes which are considered suitable for smart cards. These schemes have been already mentioned and they are the Schnorr and Fiat-Shamir algorithms. The parameters considered are the number of operations and interchanged bits (see Table 1) and the length of the keys. These parameters are directly related to the limitations of the cards in terms of memory space, computational power and slow serial $\mathrm{I} / \mathrm{O}$.

Table 1 Complexity of the new scheme

\begin{tabular}{lccc}
\hline & \multicolumn{2}{c}{ Average number of multiplications $(t=80)$} & $\begin{array}{c}\text { Number of } \\
\text { interchanged bits }\end{array}$ \\
\cline { 2 - 3 } & \multicolumn{2}{c}{ Transmission } & Reception \\
\cline { 2 - 3 } Encryption & 240 & 240 & 672 \\
Signature & 240 & 260 & 672 \\
General algorithm & 480 & $240+260$ & 1184 \\
& & & \\
\hline
\end{tabular}

Some observations must be done:

1. The number of operations can be reduced by optimization.

2. Part of the calculations in transmission can be performed in a pre-processing stage and/or using an algorithm to simulate random exponentiations, since the exponentiation is independent of the message. So, the most-time consuming operations can be precomputed in the case of the signature generation and in the encryption scheme (or in the authenticacion procedures). In the case of the general scheme, including encryption, authentication and electronic signature, one of the two exponentiations can also be precomputed.

3. Verifying the signature requires to check that $\left(z^{-e \bmod q} \cdot v_{A}^{y}\right) \bmod p=b$. It can be computed such as Schnorr shows in (Schnorr, 1991):

Initiation: $\mathrm{e}=\sum_{\mathrm{i}=0}^{79} \mathrm{e}^{\mathrm{i}} \cdot 2^{\mathrm{i}}, \mathrm{y}=\sum_{\mathrm{j}=0}^{159} \mathrm{y}^{\mathrm{j}} \cdot 2^{\mathrm{j}}$, with $\mathrm{e}_{\mathrm{i}}, \mathrm{y}_{\mathrm{j}} \varepsilon\{0,1\}$.

1. We calculate and store $\left(\mathrm{z}^{-1} \cdot \mathrm{v}_{\mathrm{A}}\right)$.

2. $x=1$.

3. $x=x^{2} \cdot z^{\text {ei }} \cdot\left(v_{A}\right)^{y i}$ for $i=159$ to 0 .

4. $1=x$.

Finally, we must indicate that the secret and public keys are 160 and 512 bits long, respectively. 


\subsection{Comparison}

The scheme presented by Schnorr is based on the discrete logarithm problem. Therefore, the components of the Schnorr algorithm and the components of the new scheme are the same. On the other hand, the protocol proposed by Fiat and Shamir is based on the difficulty of extracting modular square roots if the factorization of the modulus, $n$, is unknown. The modulus, $n \geq 2^{512}$, is the product of two secret primes $p$ and $q$. Public and secret keys are integers $v_{j}$ and $s_{j}$ (smallest square root of $v_{j}^{-1}$ ), respectively. A hash function, $h$, in $\left\{0, \ldots, 2^{\text {wk }}-1\right\}$, is used.

The complexity of the algorithms is shown in the Table 2.

Table 2 Complexity

\begin{tabular}{lcc}
\hline & $\begin{array}{c}\text { Schnorr } \\
(t=80)\end{array}$ & $\begin{array}{c}\text { Fiat-Shamir } \\
(k=9, w=8)\end{array}$ \\
\cline { 2 - 3 } $\begin{array}{l}\text { Length of the keys (bits) } \\
\text { Secret key } \\
\text { Public } \text { key }\end{array}$ & 160 & 4.608 \\
& 512 & 4.608 \\
Authentication scheme & & \\
Average number of operations & & $44+44$ \\
Number of interchanged bits & $240+260$ & 8.201 \\
& 752 & \\
Signature scheme & & $44+44$ \\
Average number of operations & & 8.201 \\
Number of interchanged bits & $240+260$ & \\
\end{tabular}

In order to compare the schemes we must take into account that the number of communication bits of the signature scheme presented in the Table 2 doesn't include the length of the signed message. Moreover, in the case of the algorithm of Schnorr, the amount of communcation for authentication can be reduced from 752 to 320 bits by using a hash function, and, on the other hand, part of the computational effort can be done in a preprocessing stage.

So, we can see that the Fiat-Shamir scheme has a very significative low number of operations, but it uses very long keys and messages.

The new cryptographic protocol and the Schnorr scheme are very similar in terms of complexity. The length of the keys is the same (quite short), and also the number of transmitted bits and the number of operations are approximately the same. Therefore, the main difference is their functionality. The new scheme, due to its versatility, can be used in many applications of security services. 


\section{CONCLUSIONS}

First of all, we can say that such as we have shown, the complexity of the new scheme is nearly equal than the complexity of the protocol proposed by Schnorr. The scheme proposed by Fiat and Shamir needs a lower number of operations but uses extremely long keys, and the total number of interchanged bits is enormous.

With the proposed scheme, the identification can be done with only a one-way transmission. This suposes that A doesn't have to wait for any message from B to generate its authentication message.

We would like to emphasize that with our scheme, the same algorithm allows to encrypt, authenticate and sign messages. With the Schnorr scheme it is possible to sign messages but, if we want to encipher this messages then another cryptosystem must be used. So, we should combine two different algorithms. With the new scheme, we are adding new features that can be very useful.

So, we have proposed a new cryptographic scheme which is efficient and flexible, and which can be used to encrypt (with simultaneous authentication), to authenticate the origin (identification), and to sign messages (totally compatible to the encryption and authentication scheme). Moreover, we have shown that the new squeme compares well, in terms of complexity, with algorithms which are considered specially suitable for smart cards. The basic features of the new scheme are:

- Flexibility.

- Most of the computational work is performed by the receiver. Moreover, part of the calculations in transmission can be done by the card in a precomputation stage, which is independent of the message.

- The length of the keys and the number of communication bits are pretty short.

Due to its characteristics the proposed algorithm can be considered particularly suited for interactions between smart cards and terminals.

Finally, we can mention that the new scheme can also be implemented using elliptic curves (Koblitz, 1987). In this case, shorter keys would be used and lower number of bits would be exchanged. Nevertheless, the number of calculations would be increased. We consider that it would be worth studying such implementation.

\section{REFERENCES}

Diffie, W. and Hellman, R. (1976) New directions in cryptography. IEEE Trans. on Inform. Theory, IT-22, 644-654.

Schnorr, C.P. (1991) Efficient signature generation by smart cards. Journal of Cryptology, 4, 161-174.

Fiat, A. and Shamir, A. (1987) How To Prove Yourself: Practical Solutions of Identifications and Signature Problems. Advances in Cryptology - Crypto'86, Lect. Notes in Comp. Science, 263, 186-194. 
ElGamal, T. (1985) A Public Key Cryptosystem and a Signature Scheme based on Discrete Logarithm. IEEE Trans. on Inform. Theory, IT-31, 469-472.

McCurley, K. (1990) The discrete logarithm problem. Cryptol. Computat. Number Theory, Proc. Symp. Appl. Math., 42, pp. 49-74.

Odlyzko, A. (1985) Discrete logarithms and their cryptographic significance. Adv. in Cryptology, Eurocrypt 84, Lect. Notes in Comp. Science, 209, 224-314.

Koblitz, N. (1987) A Course in Number Theory and Cryptography. Springer-Verlag, New York.

\section{BIOGRAPHY}

Christian Marco received the Engineer of Telecommunication degree from the Universidad Politécnica de Cataluña, Spain, in 1993. Actually he is doing his doctoral work at the Universidad Politécnica de Cataluña under Paz Morillo.

He began his academic career at the Centro Politécnico Superior de Zaragoza, Spain, as Assistan Professor in 1993. Since 1995 he is Assistant Professor at the Escuela Técnica Superior de Ingenieros de Telecomunicación de Barcelona, Spain. He has been working in the field of telematics with particular emphasis on security services in communications. His main research interest lie in the area of public key cryptography and its applications.

Paz Morillo studied mathematics at the Facultad de Matemáticas de la Universidad Autónoma de Barcelona, Spain. In 1987, she received the $\mathrm{Ph}$. $\mathrm{D}$. in computer science at the Facultad de Informática de Barcelona, Spain.

In 1985, she joined the Escuela Técnica Superior de Ingenieros de Telecomunicación de Barcelona, Spain, where she became Full Professor in 1987. She has been working in the field of applied mathematics, specially in graph theory and number theory. Her current research interest is the application of number theory in cryptology. In particular, she works in the areas of public key cryptography, secret sharing schemes and primality testing. 